\title{
Surveillance of bacterial meningitis in an Angolan pediatric hospital after the introduction of pneumococcal conjugate vaccines
}

\author{
Suvi Urtti ${ }^{1}$, Manuel L. Cruzeiro², Linda de Gouveia ${ }^{3}$, Heikki Peltola ${ }^{1}$, Anne von Gottberg ${ }^{4}$, Moe H. Kyaw $^{5}$, \\ Tuula Pelkonen ${ }^{2}$ \\ ${ }^{1}$ Children's Hospital, Helsinki University Central Hospital, Helsinki, Finland; University of Helsinki, Helsinki, Finland, 2 Hospital Pediátrico David \\ Bernardino (HPDB), Luanda, Angola, ${ }^{3}$ Centre for Respiratory Disease and Meningitis (CRDM), National Institute for Communicable Diseases (NICD), \\ division of the National Health Laboratory Service (NHLS), Johannesburg, South Africa, ${ }^{4}$ Centre for Respiratory Disease and Meningitis (CRDM), \\ National Institute for Communicable Diseases (NICD), division of the National Health Laboratory Service (NHLS), Johannesburg, South Africa; School of \\ Pathology, Faculty of Health Sciences, University of the Witwatersrand, Johannesburg, South Africa, ${ }^{5}$ Sanofi Pasteur, Swiftwater, USA ${ }^{\star \star}$ \\ Keywords: angola, bacterial meningitis, global health \\ https://doi.org/10.29392/joghr.3.e2019091
}

Journal of Global Health Reports

Vol. 3, 2019

\begin{abstract}
Background
Bacterial meningitis (BM) is a significant cause of mortality in children; with deaths from BM in children aged $<5$ years in Angola estimated at 2395 in 2015. Streptococcus pneumoniae is one causative agent for BM in young children, and Angola introduced routine immunization with a 13-valent pneumococcal vaccine (PCV13) in 2012. This study assessed BM etiology in children in Angola following introduction of PCV13.
\end{abstract}

\section{Methods}

This was a prospective, observational, single-site study conducted from October 2016 to October 2017. Children aged 3 months-15 years were included. Suspected meningitis was defined according to the World Health Organization (WHO) guidelines. Detection and characterization of $S$. pneumoniae, Neisseria meningitidis, and Haemophilus influenzae was carried out using polymerase chain reaction (PCR) assay.

\section{Results}

A total of 1732 patients were enrolled and bacteria were detected in 137 cerebrospinal fluid samples. The most common pathogen detected was $S$. pneumoniae (43/137, 31.4\%), followed by $H$. influenzae (21/137, 15.3\%) and N. meningitidis (16/137, 11.7\%) with $48 \%$ (10/ 21 ) of $S$. pneumoniae cases caused by PCV13 vaccine serotypes. $N$. meningitidis isolates were serogroups B, C and Y, and 67\% (10/15) of H. influenzae isolates were type b. In patients with meningitis and a pathogen detected, mortality (38\% (50/132) vs. $12 \%(101 /$ $818), P<0.0001)$ and severe neurological sequelae $(15 \%(10 / 67)$ vs. $5 \%(35 / 667), P=0.002)$ were higher than in those without pathogens detected.

\section{Conclusions}

These data highlight that strategies to reduce the incidence of BM caused by $N$. meningitidis, $S$. pneumoniae and $H$. influenzae must continue to reduce mortality and morbidity.

The global burden of bacterial meningitis (BM) is high, with an estimated 115,000 deaths in children aged under five years in $2015 .^{1}$ Outcomes from BM in children in Africa are often poor, with reports of mortality ranging from $1-75 \%$ depending on etiology, and the incidence of severe sequelae ranging from $3-57 \% .2,3$ The etiology of BM varies with age; although Streptococcus pneumoniae is common in all age groups, it is most prevalent in young children, Neisseria meningitidis and Haemophilus influenzae are also common in children aged under five years. ${ }^{4,5}$

Angola is amongst the most impoverished countries in Africa despite a fast growing economy. ${ }^{6}$ The under- 5 year mortality rate in the country is one of the highest in the world, 157 per 1000 live births in $2015 .{ }^{7}$ Angola has made progress in reducing BM mortality in children aged under 5 years, from 6695 estimated deaths in 2000 to an estimated 2395 deaths in 2015. However, BM still accounts for approximately $1 \%$ of the deaths in those aged under 5 years. ${ }^{1}$ Angola's routine immunizations include the pentavalent vaccine (diphtheria/tetanus/pertussis [DTP], hepatitis B, and $H$. influenzae type b) which was introduced in 2006, and the 13-valent pneumococcal vaccine (PCV13; against 13 serotypes of $S$. pneumoniae) which was introduced in 2013. Both are administered at 2, 4 and 6 months of age. Current estimates for three doses of DTP vaccine coverage in chil- 
dren in Angola range from $48 \%$ to $84 \%,{ }^{7-9}$ while estimates for PCV13 vaccination rates range from $59 \%$ to $82 \% .{ }^{9}$

Following the introduction of routine childhood pentavalent vaccination in 2006, the number of BM cases due to H. influenzae type b dropped by $86 \%$ in the country. ${ }^{10}$ There is also substantial evidence that the use of PCV vaccines can dramatically reduce cases of invasive pneumococcal disease in high-, middle- and low-income countries. ${ }^{11-17}$ This study assessed the etiology of BM in Angola in children aged 3 months to 15 years following the introduction of PCV13 vaccination.

\section{METHODS}

\section{STUDY DESIGN}

This was a prospective, observational, single site, descriptive study conducted between 21 October 2016 and 20 October 2017 at Hospital Pediátrico David Bernardino (HPDB) in Luanda, Angola, a 300-bed pediatric referral and teaching hospital.

All children aged up to 15 years admitted to HPDB and presenting with suspected meningitis were approached for enrolment and screened for study eligibility by cerebrospinal fluid (CSF) analysis. Only children aged from 3 months to 15 years were included in this analysis. Suspected meningitis was defined according to the World Health Organization (WHO) $)^{18}$ guidelines and included sudden onset of fever $\left(>101.3^{\circ} \mathrm{F}\left(38.5^{\circ} \mathrm{C}\right)\right.$ rectal or $100.4^{\circ} \mathrm{F}$ $\left(38.0^{\circ} \mathrm{C}\right)$ axillary) and one of the following signs: neck stiffness, altered consciousness with no alternative diagnosis, other meningeal signs in a child aged 30 days to $\leqslant 15$ years or a clinical diagnosis of meningitis in a hospitalized child. Macroscopic and microscopic examination of CSF was conducted at the local laboratory to determine if the case was probable or confirmed meningitis. Gram staining and cell counting was conducted on all specimens, and bacterial culture was performed if CSF leucocytes were $>10 / \mathrm{mL}$, or the glucose concentration was $<25 \mathrm{mg} / \mathrm{dL}$. Bacterial antigen test was conducted when available, if $>100$ leukocytes $/ \mathrm{mm}^{3}$ were present and bacterial culture was negative.

Probable meningitis was defined as a CSF from a suspected meningitis case characterized by turbidity or leukocytosis (>100 cells $/ \mathrm{mm}^{3}$ ), or leukocytosis (10-100 cells/ $\left.\mathrm{mm}^{3}\right)$ AND either an elevated protein $(>100 \mathrm{mg} / \mathrm{dL})$ or decreased glucose $(<40 \mathrm{mg} / \mathrm{dL})$ concentration. ${ }^{18}$

Confirmed BM was defined as a suspected BM case that was laboratory-confirmed by culture or bacterial identification (i.e. by Gram stain, antigen detection, PCR). ${ }^{18}$

CSF samples were stored and sent to the Centre for Respiratory Diseases and Meningitis (CRDM), National Institute for Communicable Diseases (NICD), Johannesburg, South Africa for polymerase chain reaction (PCR) testing.

\section{LABORATORY METHODS}

All CSF specimens were transported to the local laboratory within three hours of sampling for macroscopic and microscopic examination according to local guidelines. One sample tube containing sufficient CSF from all cases of suspected meningitis was stored at $-80^{\circ} \mathrm{C}$ as soon as possible, to protect the integrity of the bacterial DNA. Samples were shipped to NICD for real-time PCR to detect meningococcus, pneumococcus and $H$. influenzae and perform further characterization on PCR-positive samples.

DNA extraction was performed using the MagNA Pure 96 instrument (Roche, Mannheim, Germany) with Viral NA small volume kit (Roche) according to manufacturer's instructions. The molecular detection of meningitis pathogens targeted the ctrA, lytA and hpd genes for $N$. meningitidis, $S$. pneumoniae and $H$. influenzae respectively, using a multiplex real-time PCR assay as previously described (up to and including cycle threshold $\left[\mathrm{C}_{\mathrm{t}}\right]=39$ ). ${ }^{19}$ All real-time PCR assays were performed using the Applied Biosystems 7500 Fast real-time PCR platform (Applied Biosystems, Foster City, California, USA). ${ }^{19}$

All PCR positive samples were further serotyped by realtime PCR. CSF samples positive for ctrA or $h p d$ targets (up to and including $\mathrm{C}_{\mathrm{t}}=39$ ) underwent serogrouping/typing: $c t r \mathrm{~A}$ positive samples were confirmed using two multiplex reactions for detection of serogroups $\mathrm{A}, \mathrm{W}, \mathrm{X}, \mathrm{B}, \mathrm{C}$, and $\mathrm{Y},{ }^{20}$ and the serotypes in $h p d$ positive samples were determined using three multiplex reactions. ${ }^{21,22}$ lytA-positive samples with $C_{t}$ values $\leqslant 35$ were tested by eight multiplex reactions detecting 38 serotypes. ${ }^{23,24}$

\section{ENDPOINTS}

The primary objective was to describe the etiology of $N$. meningitidis, S. pneumoniae and $H$. influenzae detected in CSF samples from children hospitalized with suspected meningitis and to examine their association with patient or disease characteristics. Secondary objectives included determining the causative agents of acute pediatric BM and determining the serogroup and serotype of each case of $N$. meningitidis, $S$. pneumoniae and $H$. influenzae. Additionally, duration of hospital stay, case-fatality ratio and incidence of severe neurological sequelae were assessed throughout the study period. Severe neurological sequelae at discharge were defined as blindness, quadriplegia/paresis, hydrocephalus requiring a shunt, or severe psychomotor retardation. Information on each patient was collected including age, sex, nutritional status, vaccination history, underlying chronic illness, sickle cell status, diagnosis of present illness, dates of hospitalization and discharge/death, and outcome. The information was collected using a form prepared specifically for this study, completed by study nurses and doctors.

\section{STATISTICAL ANALYSIS}

The data were analyzed with StatView version 5.1 (SAS Institute, Cary, NC, USA). The $\chi^{2}$ and Fisher's exact test were used for comparisons of categorical variables, and MannWhitney and Kruskal-Wallis tests for continuous variables; $P$-values $<0.05$ were regarded as statistically significant. The data are presented descriptively with means, percentages, medians and interquartile ranges (IQRs) where appropriate. 


\section{STUDY CONDUCT AND ETHICAL APPROVAL}

This study was approved by the Ethics Committee of HPDB and the Hospital Director. Parents or guardians of all enrolled patients with suspected BM provided informed consent to use their child's data and samples for further analysis.

\section{RESULTS}

\section{STUDY POPULATION}

CSF samples were taken from 2573 children (aged $\leqslant 15$ years) presenting to HPDB with suspected meningitis; of these, 1732 (67\%) were aged from 3 months to 15 years (Figure S1 in the Online Supplementary Document). The demographics of these children are shown in Table S1 in the Online Supplementary Document. Reasons for lumbar puncture were provided for 1107 patients, with altered level of consciousness the main reason given (64\%, 709/1107) (Table S2 in the Online Supplementary Document). The characteristics of the CSF samples are described in Table S3 in the Online Supplementary Document. There were 196/1732 (11.3\%) cases of BM (139 (8.0\%) confirmed and 57 [3.3\%] probable).

The demographics of the children with BM are shown in Table 1. The median age was 29 months for those with confirmed or probable BM, $40 \%$ were female and $70 \%$ had complete vaccinations for their age. There was a significantly higher proportion of children with sickle cell disease in those with confirmed or probable BM (18\%) compared with those finally classified with only suspected meningitis (4\%), and there was a significantly lower proportion of children with malaria in those with confirmed or probable BM (25\%) compared with those with suspected BM (60\%).

\section{ETIOLOGY AND ORGANISM CHARACTERISTICS}

Bacteria were detected in 137/1732 (7.9\%) lumbar puncture samples by culture, PCR, latex agglutination and/or Gram staining (Table S4 in the Online Supplementary Document). CSF culture was performed in 326 cases, of which 55 (16.9\%) were positive; PCR was performed in 444 cases, of which 53 (11.9\%) were positive and 63 (14.2\%) inconclusive; latex agglutination was performed in 61 cases, of which 21 (34.4\%) were positive; and gram-staining was performed in 326 cases, of which 95 (29.1\%) were positive. The most common pathogen detected was $S$. pneumoniae $(31.4 \%, 43 / 137)$, followed by $H$. influenzae $(15.3 \%, 21 / 137)$ and $N$. meningitidis (11.7\%, 16/137) (Figure 1). Of the 1732 CSF samples collected, 444 (26\%) had sufficient CSF volume for PCR testing for the three bacterial organisms of interest. For S. pneumoniae, $10 / 21$ (48\%) of the cases were due to PCV13 vaccine serotypes (Figure 2, Panel A), for $N$. meningitidis, the cases were due to $\mathrm{B}, \mathrm{C}$ and $\mathrm{Y}$ serogroups (Figure 2, Panel B) and for $H$. influenzae, $10 / 15$ (67\%) of the samples were due to type $b$ and $4 / 15$ (27\%) to type a (Figure 2, Panel C). The median duration of hospital stay was 13 days for those with $\mathrm{BM}$, longer than for those without (Table 2). Among patients with BM with known outcomes, mortality was $38 \%$ (50/132) and severe neurological sequelae reported in 15\% of patients (10/67) (Table 2). Both were significantly higher than in those who had CSF samples and who did not have confirmed BM.

\section{DISCUSSION}

BM remains an important cause of morbidity and mortality in Luanda in the period following the introduction of the PCV13 vaccine. The mortality rate of $38 \%$ among children with BM with known outcomes was similar to that reported at the same center in 2004 (35\%). ${ }^{25}$ Severe sequelae were reported for $15 \%$, which is a reduction from that identified in 2004 (24\%). ${ }^{25}$ In the present study, the median age of children with BM was 29 months, similar to that reported in other studies of this nature in different countries. ${ }^{22,23}$ These data highlight that there is more to be done to reduce mortality due to BM, particularly in children aged under 5 years.

Prior to the introduction of the pentavalent vaccine in Angola, a study in the same hospital in Luanda in 2004, identified $H$. influenzae type $\mathrm{b}(60 \%)$ as the main cause of BM, followed by $S$. pneumoniae (24\%) and $N$. meningitidis (10\%). ${ }^{25}$ Another study at HPDB over the period 2003-2012 highlighted that the introduction of the pentavalent vaccination program reduced the number of BM cases due to $H$. influenzae type b by $86 \%$, to just $4 \%$ of cases. ${ }^{10}$ In 2004 , before the use of PCV13 in Angola, there were 93 cases of pneumococcal meningitis identified in children in HPDB, ${ }^{26}$ our one year study found 43 cases of pneumococcal meningitis, indicating a large decline of BM caused by S. pneumoniae in the post PCV13 period. However, this study still found that $S$. pneumoniae was the most common causative agent of BM, followed by $H$. influenzae and $N$. meningitidis; similar patterns have been identified in other BM surveillance studies. ${ }^{5,10,27,28}$

In our study, $70 \%$ of children were reported to have complete vaccinations for their age; the official country estimates for 2016 to 2018 were $74 \%$ to $82 \%$, with a WHO/ UNICEF estimates of $59 \%$ to $67 \%$ over the same period. ${ }^{9}$ The reason for the discrepancy between the estimates of vaccine coverage is unknown. The vaccine coverage in our study may have been higher than the WHO/UNICEF estimate as the hospital is based in an urban area which may provide better access to vaccines and health clinics than rural areas of Angola. A further consideration in this study is that the PCV13 vaccine was only introduced in 2013, and so older children would not have had the opportunity to receive this vaccine, but would still have been recorded as having complete vaccination for their age; we estimate that $40 \%$ of the children in our study would have been too old to receive PCV13 as part of their routine immunizations. Despite vaccine introduction, disease due to vaccine serotypes still occurred in this study, $48 \%$ of the $S$. pneumoniae infections were due to serotypes covered by the PCV13 vaccine routinely used in Angola, and $67 \%$ of cases due to H. influenzae were type $b$, covered by the pentavalent vaccine. Of note, there was previously no report of $H$. influenzae type a BM in Luanda, ${ }^{10,25}$ but four cases were identified in our study. Currently there is no vaccine against this serotype. In order to guide appropriate prevention strategies against $H$. influenzae disease and the development of a vaccine against 
Table 1. Demographics of children with and without confirmed or probable BM

\begin{tabular}{|c|c|c|c|c|}
\hline Characteristics & $\begin{array}{l}\text { Number } \\
\text { analyzed }\end{array}$ & $\begin{array}{c}\mathrm{BM}^{*} \\
(\mathrm{n}=196)\end{array}$ & $\begin{array}{c}\text { Suspected BM } \\
(n=1536)\end{array}$ & P-value \\
\hline \multicolumn{5}{|l|}{ Demographics } \\
\hline Age in months, median (IQR) & 1154 & $29(11-72)$ & $48(21-90)$ & 0.0001 \\
\hline Female sex, n (\%) & 1732 & $78 / 196(40)$ & $638 / 1536(42)$ & 0.64 \\
\hline Electricity at home, $\mathrm{n}(\%)$ & 734 & $88 / 101(87)$ & $567 / 633(90)$ & 0.46 \\
\hline Complete vaccinations for age, $\mathrm{n}(\%)$ & 631 & $63 / 90(70)$ & $361 / 541(67)$ & 0.54 \\
\hline \multicolumn{5}{|l|}{ History of acute illness } \\
\hline Fever in days, median (IQR) & 881 & $4(3-7)$ & $3(2-6)$ & 0.0003 \\
\hline $\begin{array}{l}\text { Previous fever and/or malaria treatment, } \mathrm{n} \\
\text { (\%) }\end{array}$ & 617 & $70 / 95(74)$ & $316 / 522(61)$ & 0.015 \\
\hline Previous antibiotics, n (\%) & 434 & $28 / 70(40)$ & $74 / 364(20)$ & 0.0007 \\
\hline \multicolumn{5}{|l|}{ Findings on admission } \\
\hline Seizures, n (\%) & 942 & $89 / 129(69)$ & $596 / 813(73)$ & 0.31 \\
\hline Altered level of consciousness, n (\%) & 954 & $94 / 129(73)$ & $609 / 825(74)$ & 0.82 \\
\hline Shock ${ }^{\#}, \mathrm{n}(\%)$ & 743 & $3 / 103(3)$ & $20 / 640(3)$ & $>0.99$ \\
\hline Dyspnea, n (\%) & 930 & $78 / 133(59)$ & $375 / 797(47)$ & 0.013 \\
\hline Dehydration, n (\%) & 886 & $38 / 125(30)$ & $147 / 761(19)$ & 0.005 \\
\hline Malnutrition, n (\%) & 908 & $42 / 128(33)$ & $164 / 780(21)$ & 0.003 \\
\hline \multicolumn{5}{|l|}{ Laboratory tests } \\
\hline Sickle cell disease, n (\%) & 742 & 19/105 (18) & $24 / 637(4)$ & $<0.0001$ \\
\hline Malaria test positive, n (\%) & 432 & $14 / 57(25)$ & $224 / 375(60)$ & $<0.0001$ \\
\hline HIV positive, n (\%) & 741 & $3 / 105$ (3) & $14 / 636(2)$ & 0.72 \\
\hline
\end{tabular}

IQR - Interquartile range, BM - bacterial meningitis

"Confirmed or probable BM. " Defined as hypotension, bradypneia, hypothermia and/or cyanose.

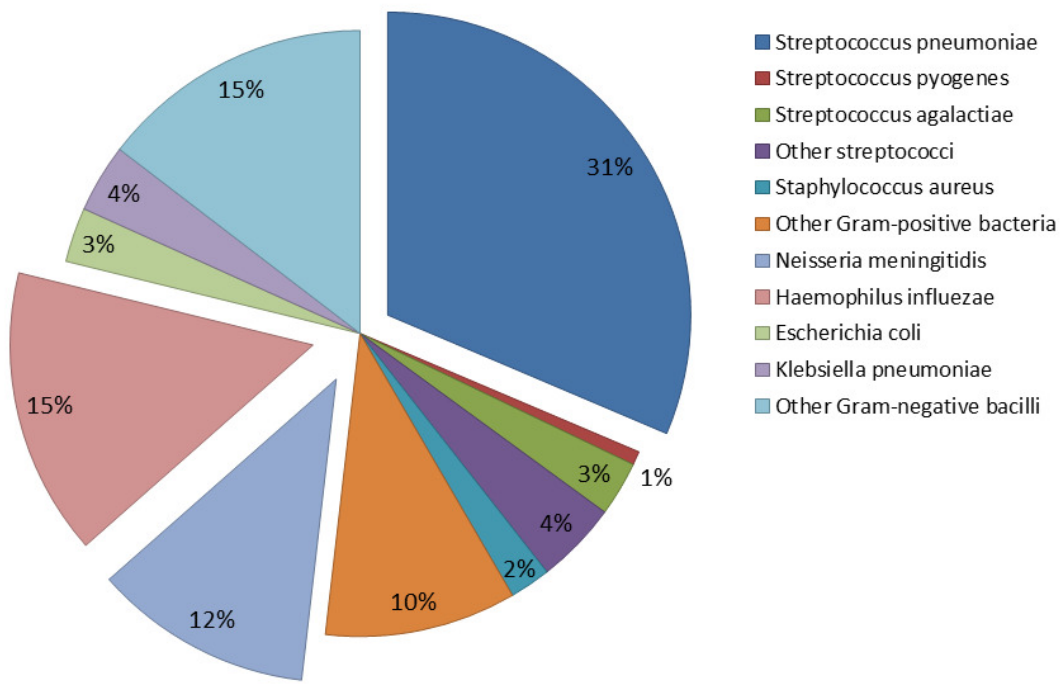

Figure 1. Breakdown by causative agent. 


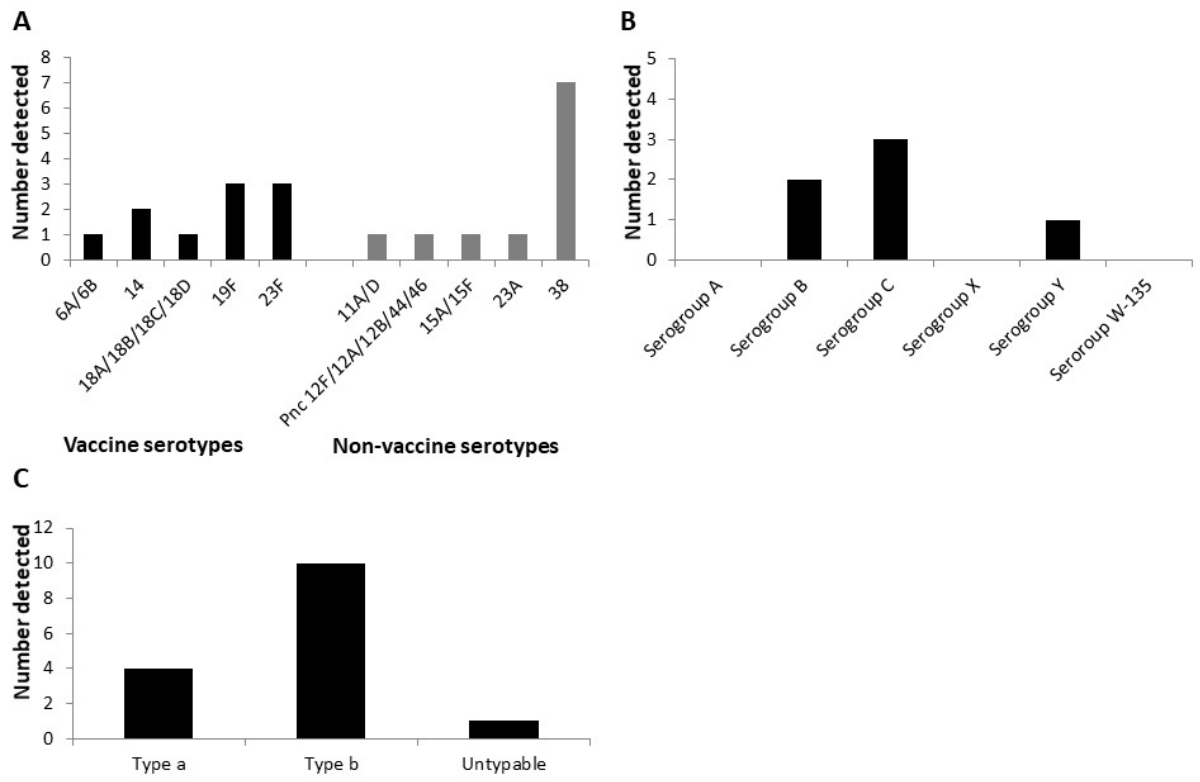

Figure 2. Serotypes for S. pneumoniae (A), N. meningitidis (B) and H. influenzae (C) detected by PCR.

Table 2. Outcomes in patients with and without confirmed or probable BM

\begin{tabular}{lcccc}
\hline Characteristics & $\begin{array}{c}\text { Number } \\
\text { analyzed }\end{array}$ & $\begin{array}{c}\text { BM }^{*} \\
(\mathbf{n}=196)\end{array}$ & $\begin{array}{c}\text { Suspected BM } \\
(\mathbf{n}=1536)\end{array}$ & P-value \\
\hline Fatal outcome, $\mathrm{n}(\%)$ & 950 & $\begin{array}{c}50 / \\
132(38)\end{array}$ & $101 / 818(12)$ & $<0.0001$ \\
\hline Length of stay, days, median (IQR) & 797 & $13(10-18)$ & $6(3-9)$ & $<0.0001$ \\
\hline Severe neurological sequelae $^{\#}, \mathrm{n}(\%)$ & 734 & $10 / 67(15)$ & $35 / 667(5)$ & 0.002 \\
\hline $\begin{array}{l}\text { Fatal outcome or severe neurological sequelae, } \\
\mathrm{n}(\%)\end{array}$ & 899 & $\begin{array}{c}60 / 121 \\
(50)\end{array}$ & $136 / 778(17)$ & $<0.0001$ \\
\hline
\end{tabular}

IQR - interquartile range, BM - bacterial meningitis

*Confirmed or probable BM. \#Defined as blindness, quadriplegia/paresis, hydrocephalus requiring a shunt, or severe psychomotor retardation.

non-type $b$ disease, continued surveillance to monitor nontype $\mathrm{b} H$. influenzae cases is being undertaken.

Causes of BM from $N$. meningitidis were also due to vaccine-preventable serogroups; however, these vaccines are not routinely used in Angola. Our study indicates that $12 \%$ of BM cases in children were caused by $N$. meningitidis. Over $50 \%$ of these meningococcal cases were in children aged $<5$ years, suggesting that vaccination in this age group could further reduce cases of $\mathrm{BM}$ in the country. Previous $N$. meningitidis outbreaks have been identified in Angola, and the introduction of targeted vaccination helped to control these. ${ }^{29}$

Strategies to reduce the incidence of BM must continue to focus on increasing vaccination coverage and raising awareness of the signs and symptoms of disease to allow for prompt medical attention and antibiotic treatment, which can help to reduce mortality and morbidity. Access to health centers that provide vaccination may be more difficult in rural areas, with potentially lower availability of vaccines, 30 so it is important to develop strategies to improve access.
The use of PCV vaccines in high-, middle- and low-income countries can reduce cases of invasive pneumococcal disease, ${ }^{11-17}$ and so efforts should focus on ensuring uptake of these vaccines. A catch-up program for vaccination of those not included in the primary series could be considered to help increase coverage. Previous studies have shown that catch-up campaigns in older children often result in better and longer-lasting protection, as immunity in infants may wane rapidly post-vaccination. ${ }^{31,32}$ Concurrent comorbidities such as HIV infection and malnutrition can also affect the severity of BM, with previous studies reporting higher mortality in populations of children affected by both BM and HIV 33 and in those infected with BM who were also found to be malnourished or underweight, ${ }^{34}$ highlighting the importance of targeting high-risk groups.

Continual surveillance of the incidence and etiology of $\mathrm{BM}$ at a city/country-wide level will also help guide prevention and control strategies in a timely and responsive manner. There are challenges to efficient surveillance programs in countries such as Angola, predominantly the lack of re- 
sources and funding to maintain such systems and conduct the necessary testing. ${ }^{35,36}$

\section{STUDY LIMITATIONS}

Findings were from a single referral hospital in Luanda, Angola. However, most children in Luanda would attend this hospital in the first instance, or be referred here by other hospitals if meningitis were suspected. The study was undertaken in an urban setting, and there are likely to be differences compared to a rural setting. The PCR assay used detected only the three pathogens ( $S$. pneumoniae, $H$. influenzae and $N$. meningitidis) and thus other bacterial causes of meningitis may have been missed. BM in neonates is known to have different etiology to BM in children, and so infants < 3 months were not included in this study; we also only included children aged up to 15 years as that is the age coverage of the hospital. Patient information for the cases was obtained from medical records, charts and data-collection forms prepared for the study and consequently were incomplete for many patients.

\section{ACKNOWLEDGEMENTS}

Editorial assistance with the preparation of the manuscript was provided by a professional medical writer, Nicola Truss $\mathrm{PhD}$ of inScience Communications, Springer Healthcare, and was funded by Sanofi Pasteur. The authors would like to thank all participants who volunteered to take part in the study; the primary investigators and their site staff.

\section{FUNDING}

This study was funded by Sanofi Pasteur. PCR at the NICD of CSF from suspected meningitis cases was funded by WHOAFRO and NICD, as part of regional reference laboratory of vaccine-preventable invasive bacterial disease (VP-IBD) surveillance responsibilities.

\section{CONFLICTS OF INTEREST}

MHK was an employee of Sanofi Pasteur when this study was conducted. SU and TP received a study grant from the Pediatric Research Foundation, Helsinki, Finland. AG reports study grants from Sanofi Pasteur not related to this work. The authors completed the Unified Competing Interest form at http://www.icmje.org/coi disclosure.pdf (available upon request from the corresponding author), and declare no further conflicts of interest.

\section{CORRESPONDENCE TO:}

Suvi Urtti
Children's Hospital
P.O. Box 347
00029 HUS
Helsinki
Finland
suvi.urtti@fimnet.fi
Tuula Pelkonen
Children's Hospita
P.O. Box 347
00029 HUS
Helsinki
Finland
tuulapelkonen@hotmail.com




\section{REFERENCES}

1. Liu L, Oza S, Hogan D, et al. Global, regional, and national causes of under-5 mortality in 2000-15: an updated systematic analysis with implications for the Sustainable Development Goals. Lancet. 2016;388(10063):3027-3035. doi:10.1016/s0140-673 6(16)31593-8

2. Edmond K, Clark A, Korczak VS, Sanderson C, Griffiths UK, Rudan I. Global and regional risk of disabling sequelae from bacterial meningitis: a systematic review and meta-analysis. Lancet Infect Dis. 2010;10(5):317-328. doi:10.1016/s1473-3099(1 0) $70048-7$

3. Ramakrishnan M, Ulland AJ, Steinhardt LC, Moïsi JC, Were F, Levine OS. Sequelae due to bacterial meningitis among African children: a systematic literature review. BMC Med. 2009;7:47. doi:10.1186/17 $\underline{41-7015-7-47}$

4. Centers for Disease Control and Prevention. Bacterial Meningitis. Accessed September 2018. http s://www.cdc.gov/meningitis/bacterial.html

5. Brouwer MC, Tunkel AR, van de Beek D. Epidemiology, Diagnosis, and Antimicrobial Treatment of Acute Bacterial Meningitis. Clin Microbiol Rev. 2010;23(3):467-492. doi:10.1128/cmr.0 0070-09

6. Republic of Angola Ministry of Health. Immunization Multi-Year Plan 2011-2015. Accessed September 2018. http://www.nationalplanningcycle s.org/sites/default/files/country docs/Angola/cmyp a ngola.pdf

7. GAVI The Vaccine Allliance. Angola. Accessed September 2018. https://www.gavi.org/country/angol a/

8. Oliveira MF, Martinez EZ, Rocha JSY. Factors associated with vaccination coverage in children $<5$ years in Angola. Rev Saúde Pública. 2014;48(6):906-915. doi:10.1590/s0034-8910.2014048 $\underline{005284}$

9. World Health Organization. Angola: WHO and UNICEF Estimates of Immunization Coverage: 2018 Revision. Accessed November 2019. https://www.who.i nt/immunization/monitoring_surveillance/data/ago.p $\underline{\mathrm{df}}$

10. Peltola H, Pelkonen T, Bernardino L, et al. Vaccine-Induced Waning ofHaemophilus influenzaeEmpyema and Meningitis, Angola. Emerg Infect Dis. 2014;20(11):1887-1890. doi:10.3201/eid201 1.140400
11. Pilishvili T, Lexau C, Farley MM, et al. Sustained Reductions in Invasive Pneumococcal Disease in the Era of Conjugate Vaccine. J Infect Dis. 2010;201:32-41. doi:10.1086/648593

12. Cohen C, von Mollendorf C, de Gouveia L, et al. Effectiveness of the 13-valent pneumococcal conjugate vaccine against invasive pneumococcal disease in South African children: a case-control study. Lancet Glob Health. 2017;5(3):e359-e369. doi:1 0.1016/s2214-109x(17)30043-8

13. von Gottberg A, de Gouveia L, Madhi SA, du Plessis M, Quan V, Soma K. Impact of conjugate Haemophilus influenzae type $\mathrm{b}$ (Hib) vaccine introduction in South Africa. Bull World Health Organ. 2006;84(10):811-818. doi:10.2471/blt.06.030361

14. von Gottberg A, de Gouveia L, Tempia S, et al. Effects of Vaccination on Invasive Pneumococcal Disease in South Africa. N Engl J Med. 2014;371(20):1889-1899. doi:10.1056/nejmoa1401914

15. Hammitt LL, Ojal J, Bashraheil M, et al. Immunogenicity, Impact on Carriage and Reactogenicity of 10-Valent Pneumococcal NonTypeable Haemophilus influenzae Protein D Conjugate Vaccine in Kenyan Children Aged 1-4 Years: A Randomized Controlled Trial. PLoS One. 2014;9:e85459. doi:10.1371/journal.pone.0085459

16. Domingues CMAS, Verani JR, Montenegro Renoiner EI, et al. Effectiveness of ten-valent pneumococcal conjugate vaccine against invasive pneumococcal disease in Brazil: a matched casecontrol study. Lancet Respir Med. 2014;2(6):464-471. $\underline{\mathrm{d}}$ oi:10.1016/s2213-2600(14)70060-8

17. Wahl B, O’Brien KL, Greenbaum A, et al. Burden of Streptococcus pneumoniae and Haemophilus influenzae type $b$ disease in children in the era of conjugate vaccines: global, regional, and national estimates for 2000-15. Lancet Glob Health. 2018;6(7):e744-e757. doi:10.1016/s2214-109x(18)302 $\underline{47-X}$

18. World Health Organization. Bacterial meningitis (including Haemophilus influenzae type b (Hib), Neisseria meningitidis, and Streptococcus pneumoniae). Accessed October 2018. http://www.wh o.int/immunization/monitoring surveillance/burden/ $\mathrm{vpd} /$ surveillance type/sentinel/meningitis surveillan ce/en/ 
19. Wang X, Theodore MJ, Mair R, et al. Clinical validation of multiplex real-time PCR assays for detection of bacterial meningitis pathogens. J Clin Microbiol. 2012;50(3):702-708. doi:10.1128/jcm.0608 7-11

20. Mothershed EA, Sacchi CT, Whitney AM, et al. Use of real-time PCR to resolve slide agglutination discrepancies in serogroup identification of Neisseria meningitidis. J Clin Microbiol. 2004;42:320-328. doi:1 $\underline{0.1128 / \mathrm{jcm} .42 .1 .320-328.2004}$

21. Wang X, Mair R, Hatcher C, et al. Detection of bacterial pathogens in Mongolia meningitis surveillance with a new real-time PCR assay to detect Haemophilus influenzae. Int J Med Microbiol. 2011;301(4):303-309. doi:10.1016/j.ijmm.2010.11.004

22. Maaroufi Y, De Bruyne JM, Heymans C, Crokaert F. Real-time PCR for determining capsular serotypes of Haemophilus influenzae. J Clin Microbiol. 2007;45(7):2305-2308. doi:10.1128/jcm.00102-07

23. Magomani V, Wolter N, Tempia S, du Plessis M, de Gouveia L, von Gottberg A. Challenges of using molecular serotyping for surveillance of pneumococcal disease. J Clin Microbiol. 2014;52(9):3271-3276. doi:10.1128/jcm.01061-14

24. Pimenta FC, Roundtree A, Soysal A, et al. Sequential triplex real-time PCR assay for detecting 21 pneumococcal capsular serotypes that account for a high global disease burden. J Clin Microbiol. 2013;51(2):647-652. doi:10.1128/jcm.02927-12

25. Pelkonen T, Roine I, Monteiro L, et al. Acute childhood bacterial meningitis in Luanda, Angola. Scand J Infect Dis. 2008;40(11-12):859-866. doi:10.108 0/00365540802262091

26. Pelkonen T, Roine I, Monteiro L, et al. Risk factors for death and severe neurological sequelae in childhood bacterial meningitis in sub-Saharan Africa. Clin Infect Dis. 2009;48(8):1107-1110. doi:10.1086/597 $\underline{463}$

27. Jayaraman Y, Veeraraghavan B, Chethrapilly Purushothaman GK, et al. Burden of bacterial meningitis in India: Preliminary data from a hospital based sentinel surveillance network. PLoS One. 2018;13(5):e0197198. doi:10.1371/journal.pone.0197 198
28. Yogev R, Guzman-Cottrill J. Bacterial Meningitis in Children. Drugs. 2005;65(8):1097-1112. doi:10.216 5/00003495-200565080-00005

29. Gaspar M, Leite F, Brumana L, Felix B, Stella AA. Epidemiology of meningococcal meningitis in Angola, 1994-2000. Epidemiol Infect. 2001;127(3):421-424. do i:10.1017/s0950268801006318

30. Frøystad M, Mæstad O, Villamil N. Health Services in Angola: Availability, Quality and Utilisation. Accessed September 2018. https://www.cmi.no/public ations/file/4319-health-services-in-angola.pdf

31. Borrow R, Abad R, Trotter C, van der Klis FRM, Vazquez JA. Effectiveness of meningococcal serogroup $C$ vaccine programmes. Vaccine. 2013;31(41):4477-4486. doi:10.1016/j.vaccine.2013.0 7.083

32. Trotter CL, Andrews NJ, Kaczmarski EB, Miller E, Ramsay ME. Effectiveness of meningococcal serogroup C conjugate vaccine 4 years after introduction. Lancet. 2004;364(9431):365-367. doi:1 0.1016/s0140-6736(04)16725-1

33. Molyneux EM, Tembo M, Kayira K, Bwanaisa L, Mweneychanya J, Njobvu A. The effect of HIV infection on paediatric bacterial meningitis in Blantyre, Malawi. Arch Dis Child. 2003;88(12):1112-1118. doi:10.1136/adc.88.12.1112

34. Roine I, Weisstaub G, Peltola H. Influence of malnutrition on the course of childhood bacterial meningitis. Pediatr Infect Dis J. 2010;29(2):122-125. 뜨 oi:10.1097/inf.0b013e3181b6e7d3

35. Dbaibo G, Tatochenko V, Wutzler P. Issues in pediatric vaccine-preventable diseases in low- to middle-income countries. Hum Vaccin Immunother. 2016;12(9):2365-2377. doi:10.1080/21645515.2016.11 $\underline{81243}$

36. Berkley JA, Mwangi I, Ngetsa CJ, et al. Diagnosis of acute bacterial meningitis in children at a district hospital in sub-Saharan Africa. Lancet. 2001;357(9270):1753-1757. doi:10.1016/s0140-6736(0 0)04897-2 